Pacific Journal of Mathematics

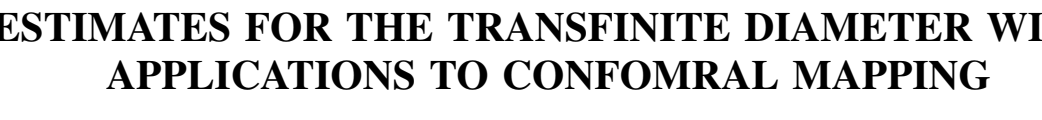




\title{
ESTIMATES FOR THE TRANSFINITE DIAMETER WITH APPLICATIONS TO CONFORMAL MAPPING
}

\author{
MeLVYN KLeIN
}

Let $f(z)$ be a member of the family $S$ of functions regular and univalent in the open unit disk whose Taylor expansion is of the form: $f(z)=z+a_{2} z^{2}+\cdots$. Let $D_{w}$ be the image of the unit disk under the mapping: $w=f(z)$. An inequality for the transfinite diameter of $n$ compact sets in the plane $\left\{T_{i}\right\}_{1}^{n}$ is established, generalizing a result of Renngli:

$$
d\left(T_{1} \cap T_{2}\right) \cdot d\left(T_{1} \cup T_{2}\right) \leqq d\left(T_{1}\right) \cdot d\left(T_{2}\right) .
$$

This inequality is applied to derive covering theorems for $D_{w}$ relative to a class of curves issuing from $w=0$, arcs on the circle: $|w|=R$ as well as other point sets.

I. Preliminary considerations.

Definition (1.1). Let $E$ be a compact set in the plane. Set:

$$
\begin{gathered}
V\left(z_{1}, \cdots, z_{n}\right)=\prod_{k>l}^{n}\left(z_{k}-z_{l}\right) \quad n \geqq 2, \quad z_{i} \in E, \\
V_{n}=V_{n}(E)=\max _{z_{1}, \cdots, z_{n} \in E}\left|V\left(z_{1}, \cdots, z_{n}\right)\right|
\end{gathered}
$$

and

$$
d_{n}=d_{n}(E)=V_{n}^{2 / n(n-1)} .
$$

The transfinite diameter of $E$ is then defined by: $d=d(E)=\lim _{n \rightarrow \infty} d_{n}$.

A full discussion of the transfinite diameter and related constants can be found in [2, Chapter 7].

The following is a theorem of Hayman [3]:

THEOREM (1.2). Suppose $f(z)$ is a function meromorphic in the unit disk with a simple pole of residue $k$ at the origin, i.e., the expansion of $f(z)$ about the origin is of the form:

$$
f(z)=\frac{k}{z}+a_{0}+a_{1} z+\cdots .
$$

Let $D_{w}$ denote the image of $|z|<1$ under the mapping $w=f(z)$ and let $E_{w}$ denote the complement of $D_{w}$ in the w-plane. Then: $d\left(E_{w}\right) \leqq k$ with equality if and only if $f(z)$ is univalent.

Using Hayman's theorem is easy to prove the following: 
THEOREM (1.3). Let $w(z)=k z+a_{2} z^{2}+a_{3} z^{3}+\cdots$ be a function univalent in $|z|<1$ and $D_{w}$ the image of $|z|<1$ under $w(z)$. Then the complement of the image of $D_{w}$ under the mapping: $\zeta=1 / w$, which we denote by $E_{\zeta}$, has transfinite diameter: $1 / k$. In particular, if $w(z)=z+a_{2} z^{2}+\cdots$ then $d\left(E_{\zeta}\right)=1$.

We will need to know the transfinite diameter of several specific sets.

Lemma (1.4). Let $E$ be the set union of:

(i) an arc of central angle $\theta, 0 \leqq \theta \leqq 2 \pi$ lying on $|w|=1$ with midpoint: $w=1$.

(ii) a linear segment $[a, b], 0 \leqq a \leqq 1 \leqq b$. Then the transfinite diameter of $E$ expressed as a function of $a, b$ and $\theta$ is given by

$$
\begin{gathered}
\cos ^{2} \frac{\theta}{4}\left[(1+b)\left(1+a^{2}-2 a \cos \frac{\theta}{2}\right)^{1 / 2}\right. \\
+(E)=\frac{\left.+(1+a)\left(1+b^{2}-2 b \cos \frac{\theta}{2}\right)^{1 / 2}\right]}{2\left[(1+a)+\left(1+a^{2}-2 a \cos \frac{\theta}{2}\right)^{1 / 2}\right]} \\
\quad \times\left[(1+b)-\left(1+b^{2}-2 b \cos \frac{\theta}{2}\right)^{1 / 2}\right]
\end{gathered}
$$

where positive roots are taken throughout.

Proof. A univalent mapping, $w=f(z)$, of $|z|<1$ onto the complement of $E$ with a simple pole at $z=0$ will be constructed. According to Theorem (1.2) the residue of the mapping function is the transfinite diameter of $E$. Define:

$$
w_{1}(z)=(z+\alpha) /(1+\alpha z)
$$

where:

$$
\begin{aligned}
& \alpha=\frac{d-c+\csc \frac{\theta}{4}}{c}-\left[\left(\frac{d-c+\csc \frac{\theta}{4}}{c}\right)^{2}-1\right]^{1 / 2}, \\
& d>1,2 c-d>0 \text {. }
\end{aligned}
$$

Define:

$$
\begin{array}{ll}
w_{2}=\frac{1}{2}\left(w_{1}+\frac{1}{w_{1}}\right) & w_{3}=c\left(w_{2}+1\right)-d \\
w_{4}=\left(w_{3}^{2}-1\right)^{1 / 2} & w_{5}=\frac{\cot \frac{\theta}{4}+w_{4}}{\cot \frac{\theta}{4}-w_{4}} .
\end{array}
$$


The composition of these five mappings is given by:

$$
w(z)=\frac{\left.\cot \frac{\theta}{4}+\left\{\frac{1}{2} c\left(\frac{z+\alpha}{1+\alpha z}+\frac{1+\alpha z}{z+\alpha}+2\right)-d\right]^{2}-1\right\}^{1 / 2}}{\left.\cot \frac{\theta}{4}-\left\{\frac{1}{2} c\left(\frac{z+\alpha}{1+\alpha z}+\frac{1+\alpha z}{z+\alpha}+2\right)-d\right]^{2}-1\right\}^{1 / 2}} .
$$

$w(z)$ maps $|z|<1$ onto the exterior of $E$ (upon proper choice of the parameters $c$ and $d$, to be made presently); it has a simple pole at the origin of residue:

$$
\frac{c}{\csc \frac{\theta}{4}+2(d-c) \sec ^{2} \frac{\theta}{4}+\tan \frac{\theta}{4} \sec \frac{\theta}{4}\left(d^{2}+1-2 c d\right)} .
$$

This is the transfinite diameter of $E$. To express it in terms of $a, b$ and $\theta$ we note that the point $w=b$ is the image of $w_{2}=1$, and the point $w=a$ is the image of $w_{2}=-1$. Using this to solve for $c$ and $d$ we find:

$$
\begin{gathered}
d=\frac{\left[a^{2}+1-2 a \cos \frac{\theta}{2}\right]^{1 / 2}}{(a+1) \sin \frac{\theta}{4}} \\
c=\frac{\left[a^{2}+1-2 a \cos \frac{\theta}{2}\right]^{1 / 2}}{2(a+1) \sin \frac{\theta}{4}}+\frac{\left[b^{2}+1-2 b \cos \frac{\theta}{2}\right]^{1 / 2}}{2(b+1) \sin \frac{\theta}{4}} .
\end{gathered}
$$

Substituting these values in the above expression for the residue we arrive at the expression given in the statement of the lemma.

When $a=b=1$ the set $E$ is simply an arc of central angle $\theta$ on the unit circle. Using the lemma we find: $d(1,1, \theta)=\sin \theta / 4$.

LEMMA (1.5). Let $E$ be the set union of two linear segments issuing from the origin at an angle $2 \pi \alpha, 0<\alpha \leqq 1 / 2$, each of length: $4 \alpha^{\alpha}(1-\alpha)^{1-\alpha}$. Then: $d(E)=1$.

Proof. The mapping of $|z|<1$ onto the exterior of $E$ is given by the Schwarz-Christoffel formula:

$$
\begin{aligned}
w & =c \cdot \int_{0}^{z} \frac{(z+1)^{1-2 \alpha}(z-1)^{2 \alpha-1}\left(z-1+2 \alpha-2\left[\alpha^{2}-\alpha\right]^{1 / 2}\right)}{\times\left(z-1+2 \alpha+2\left[\alpha^{2}-\alpha\right]^{1 / 2}\right)} \\
& =c \cdot \frac{(z+1)^{2-2 \alpha}(z-1)^{2 \alpha}}{z} .
\end{aligned}
$$


The residue of this function (the transfinite diameter of $E$ ) is $c$. Noting that the map carries $z=1-2 \alpha+2\left(\alpha^{2}-\alpha\right)^{1 / 2}$ onto $w=$ $4 \alpha^{\alpha}(1-\alpha)^{1-\alpha} e^{i \pi \alpha}$ we find that $d(E)=|c|=\left|e^{i \pi \alpha} /(-1)^{\alpha}\right|=1$.

Finally, we describe two types of symmetrization.

Steiner symmetrization of a plane set $E$ with respect to a straight line $l$ in the plane transforms $E$ into a set $E^{\prime}$ characterized by the following:

(i) $E^{\prime}$ is symmetric with respect to $l$.

(ii) Any straight line orthogonal to $l$ that intersects one of the sets $E$ or $E^{\prime}$ also intersects the other. Both intersections have the same linear measure, and

(iii) The intersection with $E^{\prime}$ consists of just one line segment, and may degenerate to a point.

Circular symmetrization of a plane set $E$ with respect to the positive real axis transforms $E$ into a set $E^{\prime}$ characterized by the following:

(i) $E^{\prime}$ is symmetric with respect to the real axis.

(ii) Any circle $|z|=r, 0 \leqq r<\infty$ that intersects one of the sets $E$ or $E^{\prime}$ also intersects the other. Both intersections have the same linear measure, and

(iii) The intersection with $E^{\prime}$ consists of just one arc with its midpoint on the positive real axis, and may degenerate to a point.

The following theorem describes the effect of these symmetrizations on the transfinite diameter $[5 ;$ p. 6 and Note A]:

THEOREM (1.6). Neither Steiner nor circular symmetrization increase the transfinite diameter.

II. Estimates for the transfinite diameter. A recent result of Renngli [6] is the following:

TheOREM (2.1). If $T_{1}$ and $T_{2}$ are compact sets in the plane, then

$$
d\left(T_{1} \cup T_{2}\right) \cdot d\left(T_{1} \cap T_{2}\right) \leqq d\left(T_{1}\right) \cdot d\left(T_{2}\right) .
$$

We will now generalize this to obtain an inequality for $n$ compact sets.

THEOREM (2.2). If $T_{1}, T_{2}, \cdots, T_{n}$ are compact sets in the plane, let $C_{k}$ be the set of all points contained in at least $k$ of the $T_{j}$ 's. Then:

$$
\prod_{k=1}^{n} d\left(C_{k}\right) \leqq \prod_{k=1}^{n} d\left(T_{k}\right) .
$$


Proof. For $n=1$ this is a triviality. For $n=2$ it is identical with Renngli's result:

$$
d\left(T_{1} \cup T_{2}\right) \cdot d\left(T_{1} \cap T_{2}\right) \leqq d\left(T_{1}\right) \cdot d\left(T_{2}\right) .
$$

Suppose the theorem is already established for $n-1$ sets. Let $B_{l k}$ be the set of all points lying in at least $k$ of the sets $T_{1}, T_{2}, \cdots, T_{n-1}$. Obviously: $B_{n-1} \subset B_{n-2} \subset \cdots \subset B_{1}$. Also:

$$
\begin{gathered}
C_{n}=B_{n-1} \cap T_{n}, \quad C_{1}=B_{1} \cup T_{n}, \\
C_{k}=B_{k} \cup\left\{B_{k-1} \cap T_{n}\right\} \quad(k=2,3, \cdots, n-1) .
\end{gathered}
$$

If $d\left(B_{n-1} \cap T_{n}\right)=d\left(C_{n}\right)=0$, (1) is certainly true.

If $d\left(B_{n-1} \cap T_{n}\right) \neq 0$, then, a fortiori,

$$
d\left(B_{k} \cap T_{n}\right) \neq 0 \quad(k=1,2, \cdots, n-1) .
$$

By (2), (3) and Renngli's inequality:

$$
\begin{gathered}
d\left(C_{n}\right)=d\left(B_{n-1} \cap T_{n}\right) \\
d\left(C_{k}\right) \cdot d\left(B_{k} \cap T_{n}\right)=d\left(C_{k}\right) \cdot d\left(B_{k} \cap B_{k-1} \cap T_{n}\right) \leqq \\
d\left(B_{k}\right) \cdot d\left(B_{k-1} \cap T_{n}\right) \\
(k=2, \cdots, n-1) \\
d\left(C_{1}\right) \cdot d\left(B_{1} \cap T_{n}\right) \leqq d\left(B_{1}\right) \cdot d\left(T_{n}\right) .
\end{gathered}
$$

Multiplying these inequalities and dividing both sides by $\prod_{k=!}^{n} d\left(B_{k} \cap T_{n}\right)$ yields

$$
\prod_{k=1}^{n} d\left(C_{k}\right) \leqq \prod_{k=1}^{n-1} d\left(B_{k}\right) d\left(T_{n}\right)
$$

and the theorem is proved, since by the induction hypothesis

$$
\prod_{k=1}^{n-1} d\left(B_{k}\right) \leqq \prod_{k=1}^{n-1} d\left(T_{k}\right)
$$

Definition (2.3). A point set $T$ will be called a broken ray provided

(i) for every $r \geqq 0$ there is a point $z \in T$ such that: $|z|=r$.

(ii) the set of numbers $r \geqq 0$ for which there is more than one point $z \in T$ such that: $|z|=r$ is a set of measure zero.

Definition (2.4). Let $T$ be a subset of a broken ray. The point sets: $\eta_{1} T, \eta_{2} T, \cdots, \eta_{n} T$ where $\left\{\eta_{k}\right\}_{1}^{n}$ are the $n$-th roots of unity, will be called symmetric images of $T$. The point set: $\left\{\mathbf{U}_{k=1}^{n} \eta_{k} \cdot T\right\}$ will be called the set of $n$-fold symmetry generated by $T$ and will be denoted by $T^{(n)}$. Subsets of $T^{(n)}$ will be denoted by $\widetilde{T}^{(n)}$. 
Definition (2.5). Let $T$ be a subset of a broken ray, $T^{(n)}$ the set of $n$-fold symmetry generated by $T$ and $\widetilde{T}^{(n)}$ a subset of $T^{(n)}$. We define the circular projection of $\widetilde{T}^{(n)}$ as a subset, $\widetilde{\tau}^{(n)}$, of the set of $n$-fold symmetry, $\tau^{(n)}$, generated by the positive real axis, $\tau$. A point $z=\eta_{k} \cdot r$ will belong to the projection $\widetilde{\tau}^{(n)}$ if and only if there is a point: $\zeta \in \eta_{k} \cdot T \cap \widetilde{T}^{(n)}$ such that $|\zeta|=r$.

Definition (2.6). Let $\widetilde{\tau}^{(n)}$ be a set such as described in definition (2.5). We will use the symbol $l_{k}$ to denote the measure of the set of real numbers $r, 0 \leqq r<\infty$ such that at least $k$ of the symmetric images of $r$ lie in $\widetilde{\tau}^{(n)}$.

REMARK (2.7). Let $L$ denote the linear measure of $\widetilde{\tau}^{(n)}$; that is, the sum of the linear measures of the $n \operatorname{legs}$ of $\tilde{\tau}^{(n)}$. Then

$$
\sum_{k=1}^{n} l_{k}=L \text {. }
$$

The reason is that if $I$ is a set of real numbers which have symmetric images on exactly $k$ legs of $\widetilde{\tau}^{(n)}$ the measure of $I$ is included in: $l_{1}, l_{2}, \cdots, l_{k}$; that is, it is counted $k$ times in: $\sum_{k=1}^{n} l_{k}$.

The following theorem of Fekete is essential to our work [2; page 259].

THeOREM (2.8). Let $E$ be a compact set and $p(z)$ a polynomial of degree $n$ :

$$
p(z)=z^{n}+c_{1} z^{n-1}+\cdots+c_{n} .
$$

Let $E_{0}$ be the set of all points $z$ such that $p(z)$ lies in $E$; we will call $E_{0}$ a root set of $E$. Then: $d\left(E_{0}\right)=d(E)^{1 / n}$.

THEOREM (2.9). Suppose $\widetilde{T}^{(n)}$ is a subset of a set of $n$-fold symmetry with: $d\left(\widetilde{T}^{(n)}\right)=1$, and $\widetilde{\tau}^{(n)}$ its circular projection. If $l_{k}(k=$ $1,2, \cdots, n)$ represent the measures defined in (2.6), then:

$$
\prod_{k=1}^{n} l_{k} \leqq 4 .
$$

Equality occurs when $\widetilde{T}^{(n)}$ is itself a set of n-fold symmetry, consisting of a single component and identical with its circular projection: $\widetilde{T}^{(n)}=\widetilde{\tau}^{(n)}$.

Proof. Let $T_{k}=\eta_{k} \cdot \widetilde{T}^{(n)},(k=1,2, \cdots, n)$. Clearly:

$$
d\left(T_{k}\right)=d\left(\widetilde{T}^{(n)}\right)=1 \quad(k=1,2, \cdots, n)
$$

since the transfinite diameter is unaffected by rigid motions. 
Let $C_{k}$ be the set of all points contained in at least $k$ of the $T_{j}$ 's; that is, the set of all points $z$ such that at least $k$ of the symmetric images of $z$ lie in $\widetilde{T}^{(n)}$. Each of the sets $C_{k}$ is a set of $n$-fold symmetry.

Let $\gamma_{k}$ be the circular projection of $C_{k}$. In view of our description of the sets $C_{k}$ it is not difficult to see that the measure of a leg of $\gamma_{k}$ is $l_{k}$.

Let $B_{k}$ be the set of which $C_{k}$ is the root set with respect to the polynomial $p(z)=z^{n}$. Since $C_{k}$ is a set of $n$-fold symmetry $B_{k}$ is a subset of a single broken ray. Let $\beta_{k}$ be the set of which $\gamma_{k}$ is the root set with respect to the polynomial $p(z)=z^{n}$. As above, $\beta_{k}$ will be a subset of a single broken ray; in this case the positive real axis.

Since $\gamma_{k}$ is the circular projection of $C_{k}$ it follows that $\beta_{k}$ is the circular projection of $B_{k}$. When $n=1$ circular projection is the same transformation as circular symmetrization. Therefore:

$$
\begin{aligned}
d\left(C_{k}\right) & =d\left(B_{k}\right)^{1 / n} \\
& \geqq d\left(\beta_{k}\right)^{1 / n} \\
& \geqq\left[\frac{\left(l_{k}\right)^{n}}{4}\right]^{1 / n}=\frac{l_{k}}{\sqrt[n]{4}}
\end{aligned}
$$

since $\beta_{k}$ has linear measure no less than: $\left(l_{k}\right)^{n}$. So finally we have:

$$
\begin{aligned}
1 & =d\left(\widetilde{T}^{(n)}\right)=\prod_{k=1}^{n} d\left(T_{k}\right) \\
& \geqq \prod_{k=1}^{n} d\left(C_{k}\right) \\
& \geqq \prod_{k=1}^{n} \frac{l_{k}}{\sqrt[n]{4}}=\frac{1}{4} \prod_{k=1}^{n} l_{k}
\end{aligned}
$$

This is the desired result: $4 \geqq \prod_{k=1}^{n} l_{k}$.

This theorem contains as a special case a result of G. Szegö [7]; in our notation his result reads: Suppose that $\widetilde{T}^{(n)}=\widetilde{\tau}^{(n)}$ (i.e., it consists of straight line segments) and that $\widetilde{T}^{(n)}$ is a connected set. Then $\prod_{k=1}^{n} L_{k} \leqq 4$ where $L_{k}$ is the linear measure of the $k$-th leg of $\widetilde{T}^{(n)}$, $(k=1,2, \cdots, n)$.

Proof. In this case: $L_{l}=l_{k}$.

The next theorem establishes bounds on the content of a set lying on a circle as a function of the radius and the transfinite diameter of the set.

TheOREM (2.10). Let $A_{1}^{\prime}, A_{2}^{\prime}, \cdots, A_{n}^{\prime}, A_{k}^{\prime} \supseteqq A_{k+1}^{\prime}$ be a nested sequence of arcs on the circle $|z|=R$ where the central angle swept out by 
$A_{k}^{\prime}$ is $\theta_{k}, \quad 0<\theta_{k} \leqq 2 \pi / n$. Let $\eta_{1}, \eta_{2}, \cdots, \eta_{n}$ denote the $n$-th roots of unity and let $\alpha(i)$ be a mapping of the set of integers $\{1,2, \cdots, n\}$ onto itself. Define:

$$
A_{k}=\eta_{\alpha(k)} A_{k}^{\prime} \quad(k=1,2, \cdots, n)
$$

and let: $A=A_{1} \cup A_{2} \cup \cdots \cup A_{n}$. Then:

$$
\prod_{k=1}^{n} \sin \frac{n \theta_{k}}{4} \leqq\left[\frac{d(A)}{R}\right]^{n^{2}}
$$

Proof. $d(A)=d\left(\eta_{k} \cdot A\right)(k=1,2, \cdots, n)$. Therefore:

$$
[d(A)]^{n}=\prod_{k=1}^{n} d\left(\eta_{k} \cdot A\right) .
$$

Let $C_{k}$ be the set of all points contained in at least $k$ of the sets: $\eta_{j} \cdot A$. It follows from our hypothesis that the sets $A_{k}^{\prime}$ are nested that:

$$
C_{k}=\eta_{1} \cdot A_{k} \cup \eta_{2} A_{k} \cup \cdots \cup \eta_{n} A_{k}
$$

for each $k, 1 \leqq k \leqq n$. Thus $C_{k}$ is the root set with respect to the polynomial $w(z)=z^{n}$ of an arc on the circle $|w|=R^{n}$ of central angle $n \cdot \theta_{k}$. The transfinite diameter of such an are is, by virtue of the equality: $d(c \cdot E)=|c| \cdot d(E)$ (c a constant) given by: $R^{n} \cdot \sin \left(n \cdot \theta_{k} / 4\right)$. Therefore by Theorem (2.8):

$$
d\left(C_{k}\right)=\left(R^{n} \cdot \sin \left(n \theta_{k} / 4\right)\right)^{1 / n} .
$$

Also, by virtue of Theorem (2.2) we have that:

$$
\prod_{k=1}^{n} d\left(\eta_{k} \cdot A\right) \geqq \prod_{k=1}^{n} d\left(C_{k}\right) .
$$

Combining inequalities (6), (7) and (8) we conclude:

$$
[d(A)]^{n} \geqq \prod_{k=1}^{n}\left[R^{n} \cdot \sin \left(n \theta_{k} / 4\right)\right]^{1 / n}
$$

or

$$
[d(A) / R]^{n^{2}} \geqq \prod_{k=1}^{n} \sin \left(n \theta_{k} / 4\right)
$$

as claimed.

III. Covering theorems. The class of functions regular and univalent in $|z|<1$ whose expansion is of the form: $f(z)=z+a_{2} z^{2}+\cdots$ will be denoted by $S$. Let $D_{w}$ be the image of the unit disk under the mapping $w=f(z) \in S$. A classical result of Koebe and Bieberbach states that $D_{w}$ contains the disk $|w|<1 / 4$ irrespective of the mapping 
function $w=f(z)$ [2; page 41]. G. Szegö later noted that [8]: If $\alpha, \beta$ are two values lying in the complement of $D_{w}$ and if the segment connecting $\alpha$ and $\beta$ passes through the origin, then: $|\alpha|+|\beta| \geqq 1$.

Generalizing these results, Michael Fekete made the following conjecture: Given $n$ rays issuing from the origin $w=0$ at equal angles $2 \pi / n$, let $L$ denote the linear measure of the intersection of these rays with $D_{w}$. Then: $L \geqq n \cdot \sqrt[n]{1 / 4}$. The theorems of Koebe-Bieberbach and Szegö are the cases $n=1$ and $n=2$. For arbitrary $n$ the inequality was proved in 1964 by Marcus [4].

Our first theorem in this section further generalizes these results by considering a more general class of curves issuing from the origin in place of the $n$ rays of Fekete's conjecture. The results of the preceding section will be used to prove this as well as various other covering theorems for the class $S$.

THEOREM (3.1). Let $f(z) \in S$ and let $D_{w}$ be the image of the disk $|z|<1$ under the mapping $w=f(z)$. Let $S^{(n)}$ be a set of $n$-fold symmetry generated by an arbitrary broken ray; $\widetilde{S}^{(n)}$, a subset of $S^{(n)}$ defined by: $\widetilde{S}^{(n)}=D_{w} \cap S^{(n)}$ and $\widetilde{\sigma}^{(n)}$ the circular projection of $\widetilde{S}^{(n)}$. Denote by $L$ the linear measure of $\tilde{\sigma}^{(n)}$. Then $L \geqq n \cdot \sqrt[n]{1 / 4}$.

Proof. Let $E_{\zeta}$ represent the image of the complement of $D_{w}$ under the transformation: $\zeta=1 / w$. Then by Theorem (1.3) it follows that: $d\left(E_{\zeta}\right)=1$. Let $T^{(n)}$ denote the set of $n$-fold symmetry that is the image of $S^{(n)}$ under the transformation $\zeta=1 / w$ and let $\widetilde{T}^{(n)}$ denote the subset of $T^{(n)}$ defined by: $\widetilde{T}^{(n)}=E_{\zeta} \cap T^{(n)}$. Denote by $\widetilde{\tau}^{(n)}$ the circular projection of $\widetilde{T}^{(n)}$. It is clear from the definition of the sets involved that $\widetilde{T}^{(n)}$ is the complement with respect to $T^{(n)}$ of the image of $\widetilde{S}^{(n)}$ under the transformation $\zeta=1 / w$ and consequently, that $\widetilde{\tau}^{(n)}$ is the complement with respect to $\tau^{(n)}=\sigma^{(n)}$ of the image of $\tilde{\sigma}^{(n)}$ under the transformation: $\zeta=1 / w$.

Let $l_{1}, l_{2}, \cdots, l_{n}$ be measures defined on $\widetilde{\tau}^{(n)}$ as in definition (2.6); let $h_{1}, h_{2}, \cdots, h_{n}$ be measures defined on $\widetilde{\sigma}^{(n)}$ in the same way. Since $d\left(E_{\zeta}\right)=1$ it follows by Theorem (2.9) that: $\prod_{k=1}^{n} l_{k} \leqq 4$. The points that contribute to the measure $l_{n-k+1}$ are points in the complement of the image of the set of points contributing to $h_{k}$ under $\zeta=1 / w$. For fixed $h_{k}$, the measure $l_{n-k+1}$ is minimized when the set whose measure is $h_{k}$ is the segment $\left[0, h_{k}\right]$ in which case: $l_{n-k+1}=1 / h_{k}$. Thus:

$$
\prod_{k=1}^{n} l_{k} \geqq \prod_{k=1}^{n} \frac{1}{h_{k}}
$$

and so:

$$
4 \geqq \prod_{k=1}^{n} \frac{1}{h_{k}} \quad \text { or: } \quad\left(\prod_{k=1}^{n} h_{k}\right)^{1 / n} \geqq \sqrt[n]{1 / 4} \text {. }
$$


Since the arithmetic mean exceeds the geometric mean:

$$
\frac{1}{n} \sum_{k=1}^{n} h_{k} \geqq \sqrt[n]{1 / 4} \text {. }
$$

According to Remark (2.7): $\sum_{k=1}^{n} h_{k}=L$, the linear measure of $\tilde{\sigma}^{(n)}$. Thus: $L \geqq n \cdot \sqrt[n]{1 / 4}$ as claimed.

THEOREM (3.2) Let $w(z) \in S$ and $D_{w}$ the image of $|z|<1$ under $w(z)$. Suppose $D_{w} \cap\{|w|=R\}$ consists of $n$ disjoint arcs $\left\{B_{k}\right\}_{1}^{n}$ where

(i) The angle subtended by the arc separating $B_{k}$ and $B_{k+1}$ is no greater than: $2 \pi / n$.

(ii) If $\left\{A_{k}^{*}\right\}_{1}^{n}$ are the $n$ arcs in the complement of $\bigcup_{k=1}^{n} B_{k}$ with respect to the circle $|w|=R$ the related set of arcs: $\left\{\eta_{k} \cdot A_{k}^{*}\right\}_{1}^{n}$ are nested.

Let the endpoints of the arc $B_{k}$ be given by: $R \cdot e^{i \theta_{2 k-1}}$ and $R \cdot e^{i \theta_{2 k}}$ $(k=1,2, \cdots, n)$.

Then:

$$
\prod_{k=1}^{n} \sin \left[n\left(\theta_{2 k+1}-\theta_{2 k}\right) / 4\right] \leqq R^{n^{2}}, \quad \theta_{2 n+1}=\theta_{1}+2 \pi .
$$

Proof. Let $A_{k}^{*}$ be the arc lying between $B_{k}$ and $B_{k+1}$. The central angle subtended by $A_{k}^{*}$ is: $\theta_{2 k+1}-\theta_{2 k}$ which by hypothesis is no greater than $2 \pi / n$. Let $A_{k}$ be the image of $A_{k}^{*}$ under the transformation $\zeta=1 / w$. The $\operatorname{arcs} A_{k}^{*}$ all lie in the complement of $D_{w}$. Hence: $A=$ $\bigcup_{k=1}^{n} A_{k} \leqq E_{\zeta}$ and so $d(A) \leqq d\left(E_{\zeta}\right)=1$. The sets $A_{k}$ lie on the circle: $|\zeta|=1 / R$. The central angle subtended by $A_{k}$ is $\theta_{2 k+1}-\theta_{2 k}$; the same as that subtended by $A_{k}^{*}$. Finally, the arcs $A_{k}$ have the nested property hypothesized for the sets $A_{k}^{*}$. Since all this is so, Theorem (2.10) is applicable; therefore:

$$
\prod_{k=1}^{n} \sin \frac{n\left(\theta_{2 k+1}-\theta_{2 k}\right)}{4} \leqq[d(A) /(1 / R)]^{n^{2}} \leqq R^{n^{2}}
$$

as claimed.

This past theorem takes no account of the fact that the complement of $D_{w}$ is a continuum containing the point at infinity. A sharpened version which takes this into account is the following:

$$
d\left(0,1, \theta_{3}-\theta_{2}\right) \cdot \prod_{k=2}^{n} \sin \frac{n\left(\theta_{2 k+1}-\theta_{2 k}\right)}{4} \leqq R^{n^{2}}
$$

where $d(a, b, \theta)$ is as defined in $\S 1$. Actually, both Theorems (3.1) and (3.2) are generalized (in a sense, combined) in the following theorem, which takes the above fact into account. The techniques used to 
prove the theorem are essentially the same as those of the foregoing proofs and so just a statement of the result will be given.

THEOREM (3.3). Let $f(z) \in S$ and $D_{w}$ be the image of $|z|<1$ under $w=f(z)$. Let $C$ be a circle of radius $R, 0<R<\infty$ and $n$ an arbitrary natural number. Let $\left\{B_{n}\right\}_{1}^{n}$ be a sequence of arcs on the circle $C$ satisfying the conditions of Theorem (3.2), $S^{(n)}$ a set of $n$-fold symmetry generated by a broken ray and $\widetilde{S}^{(n)}$ a subset of $S^{(n)}$ defined by: $\widetilde{S}^{(n)}=S^{(n)} \cap D_{w} \cap\{|w| \leqq R\}$. Let $\widetilde{\sigma}^{(n)}$ denote the circular projection of $\widetilde{S}^{(n)}$ and $\left\{h_{k}\right\}_{1}^{n}$ a sequence of measures on $\widetilde{\sigma}^{(n)}$ such as defined in definition (2.6).

Then:

$$
d\left(0,\left[\frac{R}{h_{n}}\right]^{n}, n\left[\theta_{3}-\theta_{2}\right]\right) \cdot \prod_{k=2}^{n} d\left(1,\left[\frac{R}{h_{n-k+1}}\right]^{n}, n\left[\theta_{2 k+1}-\theta_{2 k}\right]\right) \leqq R^{n^{2}} .
$$

One final application will be given.

THEOREM (3.4). Let $f(z) \in S$ and $D_{w}$ the image of the disk $|z|<1$ under $w=f(z)$. Let $L_{1}, L_{2}$ denote straight lines intersecting at $w=0$ at an angle of $\pi \alpha, 0<\alpha<1$. Let $L=L\left(D_{w} \cap\left\{L_{1} \cap L_{2}\right\}\right.$ denote the linear measure of $D_{w} \cap\left\{L_{1} \cup L_{2}\right\}$. Then:

$$
L \geqq \frac{2}{\alpha^{\alpha / 2}(1-\alpha)^{(1-\alpha) / 2}} .
$$

Proof. There is no loss in generality in assuming $L_{1}$ and $L_{2}$ are symmetric images of one-another with respect to the real axis.

A set of four points on the four legs determined by $L_{1} \cup L_{2}$, each lying at a distance $r_{0}$ from the origin, will be called a "radially symmetric set"; the points themselves will be called radially symmetric images of one-another and of the point $w=r_{0}$.

We define $h_{k}(k=1,2,3,4)$ as the measure of the set of real numbers $r, 0 \leqq r<\infty$ such that at least $k$ of the radially symmetric images of $r$ (in $L_{1} \cup L_{2}$ ) lie in $D_{w}$. Then:

$$
L\left(D_{w} \cap\left\{L_{1} \cup L_{2}\right\}\right)=\sum_{k=1}^{4} h_{k} .
$$

Map by $\zeta=1 / w$ and let $E_{\zeta}$ represent the complement of the image of $D_{w}$ under this map. Then $d\left(E_{\zeta}\right)=1$. Notice that $L_{1} \cup L_{2}$ is mappped onto itself. Let $l_{k}$ be the measure of the set of real numbers $r$ such that at least $k$ of the radially symmetric images of $r$ (in $L_{1} \cup L_{2}$ ) lie in $E_{\zeta}$. Then:

$$
\prod_{k=1}^{4} l_{k} \geqq \prod_{k=1}^{4} \frac{1}{h_{k}}
$$


Let $T_{1}=E_{\zeta} \cap\left\{L_{1} \cup L_{2}\right\}$; let $T_{2}$ be the reflection of $T_{1}$ in the imaginary axis; let $T_{3}$ be the reflection of $T_{2}$ in the real axis; let $T_{4}$ be the reflection of $T_{3}$ in the imaginary axis. Clearly:

$$
d\left(T_{1}\right)=d\left(T_{2}\right)=d\left(T_{3}\right)=d\left(T_{4}\right) .
$$

Let $C_{k}$ be the set of all points contained in at least $k$ of the $T_{j}$ 's. The set $C_{k}$ is a radially symmetric set; that is, it consists of all radially symmetric images of those points $\zeta$ such that at least $k$ of radially symmetric images of $\zeta$ lie in $T_{1}$. Thus the measure of a leg of $C_{k}$ is $l_{k}$. Let $B_{k}$ be the set consisting of four segments lying on the four rays determined by $L_{1} \cup L_{2}$, each of length $l_{k}$, the intersection of the four being the point $\zeta=0$. Since the shift of segments that transforms $C_{k}$ into $B_{k}$ can only bring extremal points closer together, it follows that: $d\left(C_{k}\right) \geqq d\left(B_{k}\right)$. Using the mapping lemma (1.5) and Fekete's theorem (2.8) the transfinite diameter of $B_{k}$ can be calculated:

$$
d\left(B_{k}\right)=\frac{l_{k}}{2 \alpha^{\alpha / 2}(1-\alpha)^{(1-\alpha) / 2}} .
$$

We have

$$
\begin{array}{rlrl}
1 & =d\left(E_{\zeta}\right) \geqq d\left(T_{1}\right) & \text { since: } T_{1} \subseteq E_{\zeta} \\
& =\left[\prod_{k=1}^{4} d\left(T_{k}\right)\right]^{1 / 4} \geqq\left[\prod_{k=1}^{4} d\left(C_{k}\right)\right]^{1 / 4} & \text { by Theorem } \\
& \geqq\left[\prod_{k=1}^{4} d\left(B_{k}\right)\right]^{1 / 4}=\left[\prod_{k=1}^{4} \frac{l_{k}}{2 \alpha^{\alpha / 2}(1-\alpha)^{(1-\alpha) / 2}}\right]^{1 / 4} \\
& \geqq \frac{1}{2 \alpha^{\alpha / 2}(1-\alpha)^{(1-\alpha) / 2}}\left[\prod_{k=1}^{4} \frac{1}{h_{k}}\right]^{1 / 4} & \frac{1}{\sum_{k=1}^{4} h_{k}}
\end{array}
$$

since the arithmetic mean exceeds the geometric mean;

$$
=\left[2 /\left(\alpha^{\alpha / 2}(1-\alpha)^{(1-x) / 2}\right)\right] \cdot(1 / L) .
$$

This sequence of inequalities means:

$$
L \geqq\left[2 /\left(\alpha^{\alpha / 2}(1-\alpha)^{(1-\alpha) / 2}\right)\right] .
$$

REMARK. When $\alpha=1 / 2$ that is, when $L_{1} \cup L_{2}$ is a set of 4 -fold symmetry, the result of the theorem reads: $L \geqq 2 /(1 / 4)^{1 / 4}=4(1 / 4)^{1 / 4}$ in agreement with Theorem (3.1).

I am grateful to the referee for supplying an abbreviated proof for Theorem (2.2). 


\section{REFERENCES}

1. M. Fekete, Über der Verteilung der Wurzeln bei gewissen algebraischen Gleichungen mit ganzzahligen Koeffizienten, Math. Z. 17 (1923), 228-249.

2. G. M. Golusin, Geometrische Funktionentheorie, Veb. Deutscher Verlag der Wissenschaften, Berlin, (1957).

3. W. K. Hayman, Some applications of the trans finite diameter to the theory of functions, J. Analyse Math. 1 (1951), 155-159.

4. M. Marcus, Transformations of domains in the plane and applications in the theory of functions, Pacific J. Math. 14 (1964), 613-626.

5. G. Polya, and G. Szegö, Isoperimetric Inequalities in Mathematical Physics, Princeton, 1951.

6. H. Renngli, An inequality for logarithmic capacities, Pacific J. Math. 11 (1961), 313-314.

7. G. Szegö, On a certain kind of symmetrization and its applications, Ann. Math. Pura Appl. (4) 40 (1955), 113-119.

8. — Jber Deutsch. Math.-Verein. 32 (1923), 45.

Received August 22, 1966. This research was supported by the National Science Foundation under research grant NSF-G24469 with the University of Maryland. The paper is a part of the author's dissertation, written under the direction of Professor Mishael Zedek.

NEW YORK UNIVERSITY

UNIVERSITY HEIGHTS 



\section{PACIFIC JOURNAL OF MATHEMATICS}

\section{EDITORS}

\section{H. SAMELSON}

Stanford University

Stanford, California

J. P. JANS

University of Washington

Seattle, Washington 98105

\section{J. DugundJI}

University of Southern California Los Angeles, California 90007

RICHARD ARENS

University of California

Los Angeles, California 90024

\section{ASSOCIATE EDITORS}

E. F. BECKENBACH
B. H. NeUmanN

\section{SUPPORTING INSTITUTIONS}

UNIVERSITY OF BRITISH COLUMBIA CALIFORNIA INSTITUTE OF TECHNOLOGY

UNIVERSITY OF CALIFORNIA

MONTANA STATE UNIVERSITY

UNIVERSITY OF NEVADA

NEW MEXICO STATE UNIVERSITY

OREGON STATE UNIVERSITY

UNIVERSITY OF OREGON

OSAKA UNIVERSITY

UNIVERSITY OF SOUTHERN CALIFORNIA

\author{
STANFORD UNIVERSITY \\ UNIVERSITY OF TOKYO \\ UNIVERSITY OF UTAH \\ WASHINGTON STATE UNIVERSITY \\ UNIVERSITY OF WASHINGTON \\ AMERICAN MATHEMATICAL SOCIETY \\ CHEVRON RESEARCH CORPORATION \\ TRW SYSTEMS \\ NAVAL ORDNANCE TEST STATION
}

Mathematical papers intended for publication in the Pacific Journal of Mathematics should be typewritten (double spaced). The first paragraph or two must be capable of being used separately as a synopsis of the entire paper. It should not contain references to the bibliography. Manuscripts may be sent to any one of the four editors. All other communications to the editors should be addressed to the managing editor, Richard Arens at the University of California, Los Angeles, California 90024.

50 reprints per author of each article are furnished free of charge; additional copies may be obtained at cost in multiples of 50 .

The Pacific Journal of Mathematics is published monthly. Effective with Volume 16 the price per volume ( 3 numbers) is $\$ 8.00$; single issues, $\$ 3.00$. Special price for current issues to individual faculty members of supporting institutions and to individual members of the American Mathematical Society: $\$ 4.00$ per volume; single issues $\$ 1.50$. Back numbers are available.

Subscriptions, orders for back numbers, and changes of address should be sent to Pacific Journal of Mathematics, 103 Highland Boulevard, Berkeley 8, California.

Printed at Kokusai Bunken Insatsusha (International Academic Printing Co., Ltd.), 7-17, Fujimi 2-chome, Chiyoda-ku, Tokyo, Japan.

\section{PUBLISHED BY PACIFIC JOURNAL OF MATHEMATICS, A NON-PROFIT CORPORATION}

The Supporting Institutions listed above contribute to the cost of publication of this Journal, but they are not owners or publishers and have no responsibility for its content or policies. 


\section{Pacific Journal of Mathematics}

\section{Vol. 22, No. $2 \quad$ February, 1967}

Paul Frank Baum, Local isomorphism of compact connected Lie groups ....

Lowell Wayne Beineke, Frank Harary and Michael David Plummer, On the

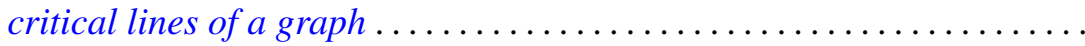

Larry Eugene Bobisud, On the behavior of the solution of the telegraphist's equation for large velocities .......................... 213

Richard Thomas Bumby, Irreducible integers in Galois extensions . . . . . . 221

Chong-Yun Chao, A nonimbedding theorem of nilpotent Lie algebras ..... 231

Peter Crawley, Abelian p-groups determined by their Ulm sequences ...... 235

Bernard Russel Gelbaum, Tensor products of group algebras ........... 241

Newton Seymour Hawley, Weierstrass points of plane domains .......... 251

Paul Daniel Hill, On quasi-isomorphic invariants of primary groups . . . . . 257

Melvyn Klein, Estimates for the transfinite diameter with applications to confomral mapping ................................ 267

Frederick M. Lister, Simplifying intersections of disks in Bing's side approximation theorem ............................. 281

Charles Wisson McArthur, On a theorem of Orlicz and Pettis ........... 297

Harry Wright McLaughlin and Frederic Thomas Metcalf, An inequality for

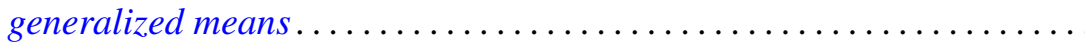

Daniel Russell McMillan, Jr., Some topological properties of piercing

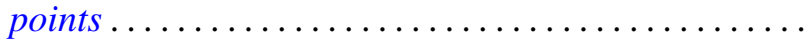

Peter Don Morris and Daniel Eliot Wulbert, Functional representation of topological algebras .

Roger Wolcott Richardson, Jr., On the rigidity of semi-direct products of Lie algebras..................................

Jack Segal and Edward Sandusky Thomas, Jr., Isomorphic

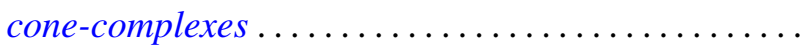

Richard R. Tucker, The $\delta^{2}$-process and related topics.... 